\title{
A new model to express and capture the design rationale in the documents
}

\author{
Jihong Liu ${ }^{1, *}$, Jiaji Wang ${ }^{1}$, and Kejian Wang ${ }^{1}$ \\ ${ }^{1}$ School of Mechanical Engineering and Automation, Beihang University, 100191 Beijing, China
}

\begin{abstract}
As important design knowledge, design rationale (DR) knowledge plays an important role in design analysis, design reasoning and design innovation. There are generally two sources of DR knowledge, one is to capture DR in the design process, and the other is to extract DR from historical design documents, but the latter is always ignored. DR model is the foundation of DR. This paper aims at the deficiency of the previous DR model and requirements for DR knowledge acquisition from design documents. We propose design rationale knowledge hierarchy (DRKH) model. The model has three layers, design intent layer, design decision layer and design basis layer. The model also has three relationships, decomposed-into, achieved-by, refer-to. Using the DRKH model, we build the algorithm framework for extracting DR from design documents. Finally, we validate the feasibility of our model by extracting DR model from a welding robot design manual.
\end{abstract}

\section{INTRODUCTION}

Product design is the process of converting a person's purpose or need into a specific physical or tool. It is a process of expressing the plan, tentative idea, and problem solving method in an ideal form, through specific operations. DR knowledge is used to represent why a product or part of a product, is designed the way it is $[1,2]$. Therefore, it is a good way to express the design process of product with DR knowledge. DR knowledge includes all relevant knowledge of product design, such as design intent decomposed for completing the product, design option made for completing the design intent, discussion of design decisions in the design process, reasons behind the design decisions, and so on [3].

In view of the importance of DR knowledge, many studies are devoted to establish DR knowledge base, i.e. express and store the acquired DR knowledge in a model and graphical language. The establishment of DR knowledge base consists of two processes: DR knowledge acquisition and DR knowledge representation. There are two sources of DR knowledge acquisition, one is stored the designer's thoughts about product design during the design process; the other is extracted DR knowledge from structured documents. At present, most of the studies are committed to capture DR knowledge during the design process, while ignoring the structured design document which as an important source of DR knowledge acquisition. This resulted in a great deal of waste of DR knowledge. If there is an access method that can effectively extract DR knowledge from design documents, it will play a significant role in promoting the establishment of DR knowledge base.
Since 1980s, many studies have made progress in the acquisition, expression and application of DR knowledge, and have made rapid progress in the DR system, however, there are still some problems in DR knowledge representation models. Taking into account the above DR knowledge acquisition source and the DR knowledge expression model problems, this paper proposes a new DR model for DR knowledge representation, which aims at extracting the DR knowledge in design documents. Section 2 introduces previous studies on DR and text processing. Section 3 presents our design rationale knowledge hierarchy (DRKH) model. In Section 4, the design specification of welding robot is taken as an example, demonstrate the process of extracting DR knowledge from a design document and expressing it with DRKH model. Section 5 concludes.

\section{RELATED WORK}

\subsection{DR representation model}

A clear and well-formed DR expression model is not only helpful for designers to understand and use the DR knowledge, more conducive to the effective reuse of design knowledge. DR models are generally divided into two categories, one is the argumentation-based DR representation model, and the other is the intention-Driven DR representation model. At present, most DR expression models belong to argumentation-based representation model. Argumentation-based DR representation model usually regards the design process as a problem solving process,

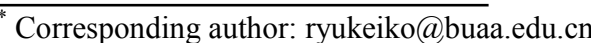


and considers that the design process is composed of multiple sub processes: 1) identify design problems; 2) propose design alternative solutions to specific problems;3) form argument that supports or opposes certain design schemes;4) the decision to adopt or reject an alternative. Therefore, argumentation-based DR representation model research focuses on the process of design decision generation. It records the concrete formation process of design decision in detail with the three elements of problem, plan and argument.

The argumentation-based representation model is represented by IBIS (issue-based information system) which is the most mature expression in the field of DR . Many teams have developed graphical DR systems based on the IBIS model for the DR knowledge management. For example, graphical IBIS (gIBIS) is the first graphical IBIS tool, and Design Rationale editor (DRed) is a more mature IBIS tool at the moment [4]. McCall suggested the Procedural Hierarchy of Issues (PHI) model, which simplifies the connection form in IBIS and makes up for the lack of dependencies between design issues [5]. Liu et al. proposed an issue, solution and artifact layer (ISAL) representation model for DR capture from patent documents [7]. It extends the basic structure of IBIS and maps the expression of design thinking process to related product components, thus making up for the shortcomings of previous argumentation-based representation model.

The intention-Driven DR representation model believes that the designer's intention will interact with the environment and evolve with it, and hope to preserve the design history by recording the evolution path of design intention. A design intent modeling system developed by Arai et al. to describe the design thinking process through intention, operation, and design flow. Ganesha utilize the design intent continually evaluated and refined to record the design process [8]. In recent years, more and more researchers have realized that the design activity is essentially an evolving cognitive process, and the intention-Driven DR representation model can reflect the cognitive characteristics of the design process more accurately.

\subsection{Document processing}

Liu had an analysis of organized text retrieval technology [9]. They studied how to advance information admittance performance in document management. Li and Ramani proposed to use shallow natural language processing build an organized and semantics-based representation from design documents [10]. Romanowski and Nagi put forward a method to making generic bills of materials to backing varied design activities [11].

From the literature review above, we can see that there is still little research on capturing DR knowledge in structured design documents. Most of the existing DR system is used to capture the DR knowledge in the design process, or for the DR knowledge retrieval, however, there are few systems for DR knowledge capture in design documents. We consider that the captured document should not only have patent document, it should involve all structured design documents. Meanwhile, captured DR knowledge should be graphical representation, so that designers can quickly understand. Therefore, a new DR knowledge representation model is proposed to support the capture of DR knowledge in a variety of structured design documents, and express the DR knowledge in a graphical way, such a model is beneficial to the management and reuse of DR knowledge in subsequent design.

\section{Design rationale knowledge hierarchy model}

\subsection{DRKH model for DR representation}

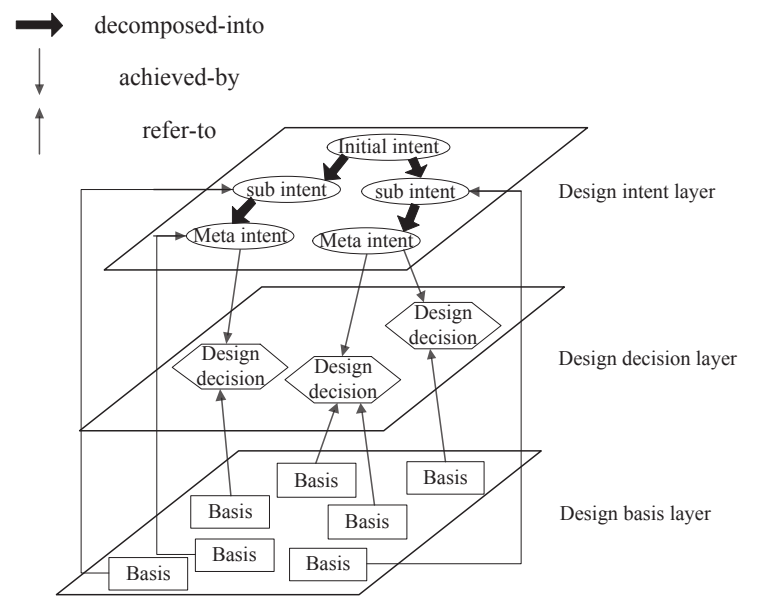

Fig. 1. Design rationale knowledge hierarchy model

The IBIS model consists of three types of elements, namely, issues, positions, arguments, and includes the relationships between the three elements. The issues are the discussion and reflection that needs to be done to complete a design goal, i.e., know how. Positions mean the method of solving the issues, that is, know how. Arguments stand for support or opposition to positions, that is, know why. They are connected by different rules to form an issue net. From the IBIS contains three elements can be seen IBIS model out of the product itself, it lacks the operation of the product. The net of issues formed by IBIS only solves an issue but there is no connection between them, and they are independent of each other. Although there are some problems in the IBIS model, it can still provide a basis for our model. Our DR knowledge model is mainly applied to capture DR knowledge in design documents, so we primarily consider three aspects in designing model:

1) The hierarchy of the model should conform to the DR structure in the design document. DR knowledge is generally composed of design issues, design options, design decisions made by the design option, design basis and design operations, but considering that the written of the design document is not record all the thoughts in the 
designer's mind, so the design options can be removed from the model.

2) There should be a directional relationship between the layers of the model, and the meaning of each direction is formulated. The DR knowledge captured in product design has a huge structure, and must have clear direction and rules, in order to achieve efficient management and reuse of DR knowledge.

3) The model needs to be graphical. Compared to a large number of texts, graphic language allows designers to quickly understand the content expressed by Graphical DR models.

According to the problems discussed above, we propose a design rationale knowledge hierarchy (DRKH) model, which is shown in Fig.1. The model consists of three layers: design intent layer, design decision layer and design basis layer.

\subsubsection{Design intent layer}

Design intent is the goal and plan for the designer to design, it can explain the factors that affect the designer's problem solving, decision making and operation execution in the design process. Design intent is both a motivation for triggering designer thinking, and sometimes a direction for designers to think about development. Design intent can be decomposed into multiple levels of sub intent, when the realization of all sub intents, the parent's intent can achieve. The intents no need to re-decompose and can implement directly, called meta intent [12].

The design intent layer usually exists as a tree structure, as shown in Fig.2.The "Welding robot design" is initial intent; "Arm structure design", "Actuator design", "Control system selection", "Wrist structure design", "Select Transmission scheme", "Select degrees of freedom and coordinate system" are sub intent; "Arm structure selection", "Hydraulic cylinder structure"," Drive structure design"," Hydraulic cylinder head screw selection"," Hydraulic cylinder selection" are meta intent.

\subsubsection{Design decision layer}

Design decision refers to the designer referring to the relevant basis, according to the evaluation criteria, put forward, analyse and compare a number of design options, and get the final solution to achieve the design intent. Design decision refers to the designer referring to the relevant basis, according to the evaluation criteria, put forward, analyze and compare a number of design options, and get the final solution to achieve the design intent.

\subsubsection{Design basis layer}

The design basis is used to explain the reasons for decision making, including the basis, standards, and tradeoffs of the designer in terms of his own expertise, experience, preferences, and situational information. In the design document, feature of the sentence which as the design basis is very obvious, they usually have the words "because", "so", "therefore", "because", etc., which express the cause. The design basis layer is an important level to embody the value of DR knowledge, whether it is the basis of intent decomposition or the basis of choosing design decision, it is an important part of the designer's thinking about the design.

\subsubsection{Connections between layers}

In the model in Fig.1, we define three relationships respectively as, "decomposed-into"," achieved-by"," refer-to". Among the relationships, decomposed-into<design intent1, design intent2> represents intent 2 is the sub intent of intent1; achieved-by<intent, decision $>$ indicates that the decision can satisfy design intent; refer-to<basis, decision $>$ indicates that the basis is the reason for choosing the decision, refer-to $<$ basis, intent $>$ represents the reason why the intent is decomposed.

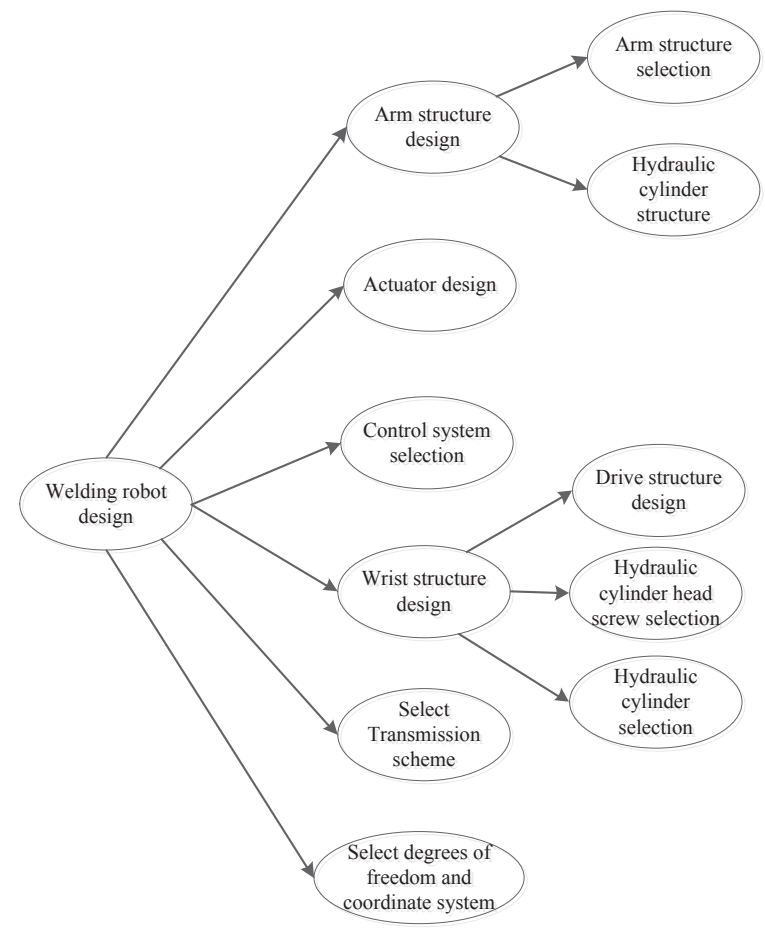

Fig. 2. Design intent tree structure

\subsection{DRKH model for DR Extraction}

\subsubsection{Design intent extraction}

Our model is applied in structured design documents to extract DR knowledge. The general form of design documentation is WORD and PDF, and structured design documents have header formats. Through extensive research, we find that the title of the design document is actually the source of the design intent. But not all titles can be used as design intent, so we will remove the title which is can not be the design intent. 
The specific plan implementation framework is shown in Fig.3.

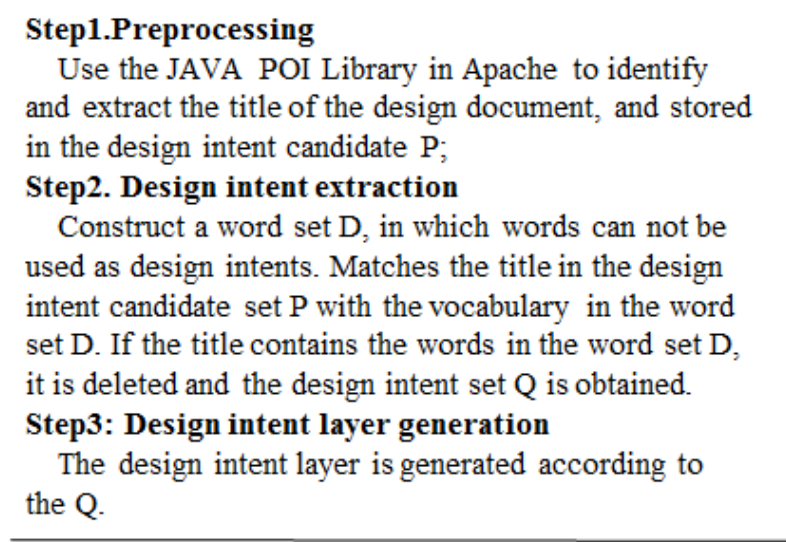

Fig. 3. The specific plan implementation framework of design intent extraction

\subsubsection{Design decision extraction}

The design decision knowledge in the design document has a characteristic, in order to realize the design intent, the design decision is usually a verb plus noun phrase. In this paper, phrase structure based syntactic analysis is applied to extract verb object phrases from design decisions. The specific plan implementation framework is shown in Fig.4.

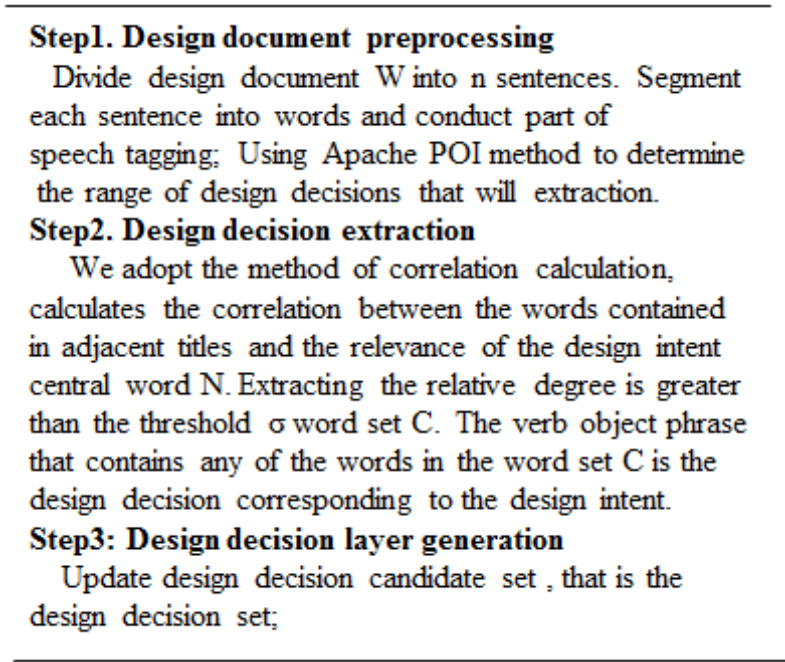

Fig. 4. The specific plan implementation framework of design decision extraction

\subsubsection{Design basis extraction}

In the above analysis of the design basis, we refer to, design basis sentence usually contain "because......","as a result of......","on the basis of......" and other explanatory words and phrases. Therefore, we can establish a lexical library for expressing the reasons, and extract the words from the vocabulary library to build the design base layer. In this process, we draw on the related algorithms of text mining. The specific plan implementation framework is shown in Fig.5.

\section{Step1. Design document preprocessing}

Divide design document $\mathrm{W}$ into $\mathrm{n}$ sentences. Segment each sentence into words and conduct part of speech tagging. Establish a design basis vocabulary library $\mathrm{O}$, then enter all the words in the dictionary that express explain and reason.

\section{Step2. Design basis extraction}

According to vocabulary that stored in the design basis vocabulary $\mathrm{O}$ extract the sentences as the design basis candidate set $\mathrm{L}$. Constructing semantic syntactic graph $\mathrm{G}(\mathrm{B}, \mathrm{X})$, The node $\mathrm{B}$ is defined as the vertex, and the connection weight is represented by the matrix $\mathrm{X}$ representing the semantic relevance of the node. The design decision and sentence in $\mathrm{L}$ is node $\mathrm{B}$. Calculate the weight between the design decision and the design basis.

\section{Step3: Design basis layer generation}

For each design decision take weight value in the former $\mathrm{k} \%$ of the design basis, and build connection.

Fig. 5. The specific plan implementation framework of design basis extraction

\section{Example using design document}

We use design document as our research data because design documents are important source of DR knowledge, and very easy to get. Fig. 6 shows the DR knowledge model extracted from the welding robot design manual using the DRKH model.

In order to verify the applicability of the proposed model, we have done two experiments. The experiment is to let different designers to extract DR knowledge from the same design document with DRKH model. Before the designers use the model, we trained them in a unified way. We analysed the DR model of 30 designers, the 30 models are extracted from the same design document. The results are shown in Table 1.

Table 1. Model similarity data

\begin{tabular}{|l|c|}
\hline \multicolumn{1}{|c|}{ Layer } & Similarity \\
\hline design intent layer & $99 \%$ \\
\hline design decision layer & $85 \%$ \\
\hline design basis layer & $81 \%$ \\
\hline
\end{tabular}

From the data we can see that the designers are almost identical in their extraction of design intent, but the similarity that extraction design decisions and the design criteria is relatively low.

\section{Conclusion}

DR model is the foundation of DR. A good DR knowledge model is not only of great help to DR knowledge acquisition, moreover, it is of great significance for the efficient use of DR knowledge. Therefore, this paper aims at the deficiency of the previous DR model and requirements for DR knowledge acquisition from design documents. We propose design 
rationale knowledge hierarchy model. The model has three layers, design intent layer, design decision layer and design basis layer. The model also has three relationships, decomposed-into, achieved-by, refer-to.

From the data in Table 1, we can see that our model needs to be improved in terms of the design decision layer and the design base layer expression. In the following research, we will focus on the improvement of the model and achieve in the design document to extract the DR knowledge.

\section{Acknowledgements}

This work has been supported by Project of National Science Foundation of China through approval No.51475027 and Project of National Key Technology R\&D Program through approval No. 2015BAF18B01.

\section{References}

1. J. Lee, K. Lai, What's in design rationale. Human-Computer Interaction, 6 (3) :251-280 (2011)

2. J. Lee, Design rationale systems: understanding the issues. IEEE Expert, 12 (3) :78-85 (1997)

3. J. Lee, The 1992 Workshop on Design Rationale Capture and Use, AI Mag., 14(2) :24-26 (1993)

4. J. Conklin, M. Begeman, A Hypertext Tool for Exploratory Policy Discussion, ACM Transactions on Office Information Systems, 6(4): 301-331 (1988)
5. Fischer G., Lemke A., McCall R., et al., Making Argumentation Serve Design, Human-Computer Interaction, 6 (3) :393-419 (1996)

6. Liu Y., Liang Y., Kwong C. K., et al., A New Design Rationale Representation Model for Rationale Mining, Journal of Computing and Information Science in Engineering, 10(3): 1-10 (2010)

7. Ganeshan R., Garrett J., Finger S. A framework for representing design intent, Design Studies, 15(1): 59-84 (1994)

8. Feldman, R., Text Mining: Theory and Practice, (1988)

9. Liu, S., McMahon, C. A., and Culley, S. J., A Review of Structured Document Retrieval (SDR) Technology to Improve Information Access Performance in Engineering Document Management, Computers in Industry, 59 (1) :3-16 (2008)

10. Li, Z., and Ramani, K., Ontology-Based Design Information Extraction and Retrieval, Artif. Intell. Eng. Des. Anal. Manuf., 21 (2) :137-154 (2007)

11. Romanowski, C. J., and Nagi., A Data Mining Approach to Forming Generic Bills of Materials in Support of Variant Design Activities, ASME J.Comput. Inf. Sci. Eng., 4 (4) :316-328 (2004)

12. Liu J. H., Zhan H. F., A reconstruction method of the design rationale model based on design context, (2013)

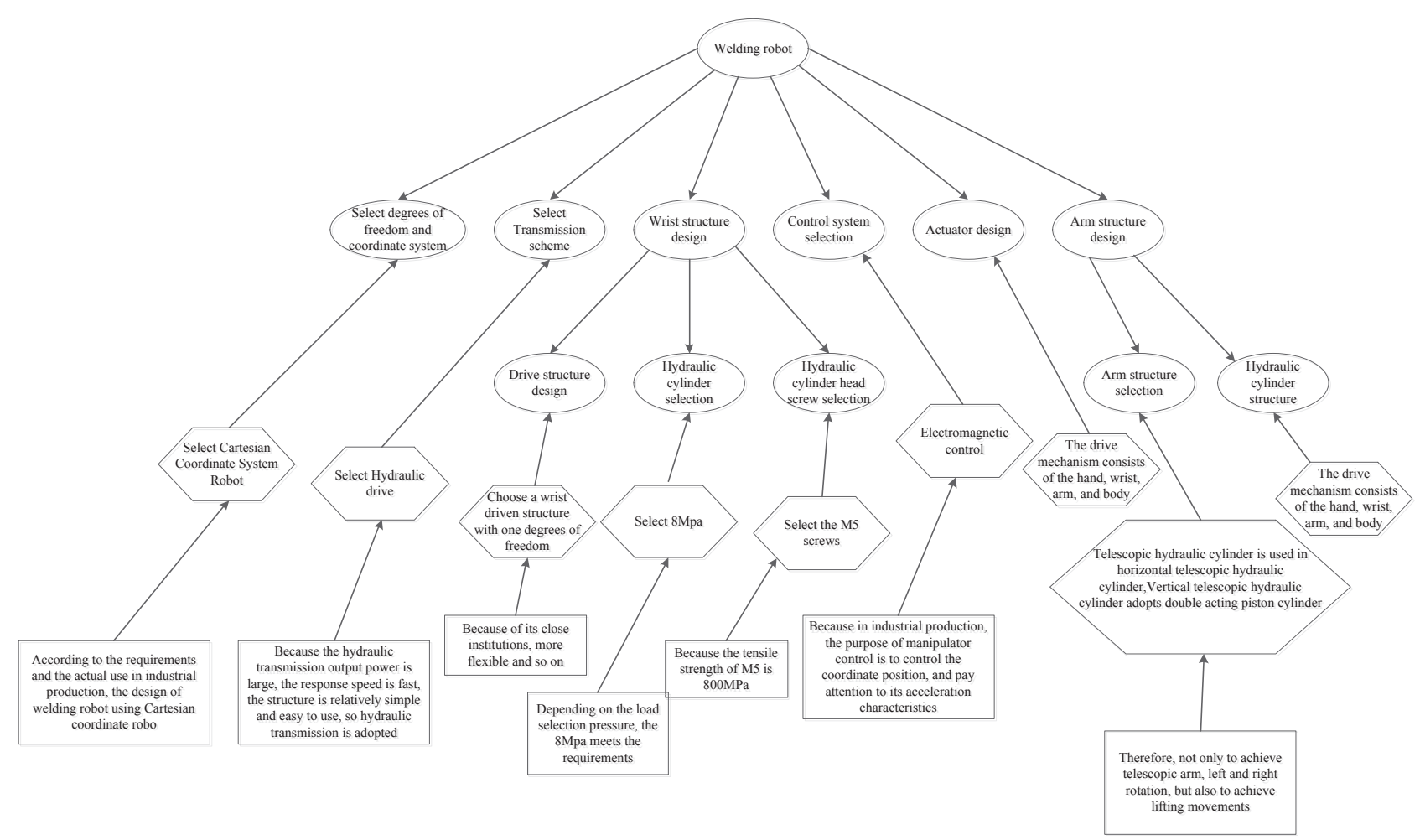

Fig. 6. The DR knowledge model extracted from the welding robot design manual 